Tohoku J. exp. Med., 1970, 101, 363-374

\title{
Arteriographic Studies of Splenic and Hepatic Arteries in Portal Hypertension
}

\author{
Hidemi Yamauchi, Yasuo Suda, Kyoji Yamamoto and \\ Toshio Sato \\ Department of Surgery, Tohoku University School of Medicine, \\ Sendai
}

Yamauch, H., Suda, Y., Yamamoto, K. and Sato, T. Arteriographic Studies of Splenic and Hepatic Arteries in Portal Hypertension. Tohoku J. exp. Med., 1970, 101 (4), 363-374 — The radii of the splenic and proper hepatic arteries were estimated by means of selective celiac arteriography in cases of portal hypertension and of splenomegaly without portal hypertension. The results were evaluated in comparison with normal arteriograms. The estimated radii of the splenic arteries were in satisfactory agreement with the histometrical ones. It was concluded that the splenic and hepatic arterial flow could be proportional to the 2.7th power of the radii of the respective arteries on arteriograms, as in the histometrical study.1,2 In cases of portal hypertension and appreciable increase in the radius of the splenic artery was demonstrated. In splenomegaly without portal hypertension, as in idiopathic thrombocytopenic purpura, hemolytic jaundice, such an increase in the radius of the splenic artery was not observed. In portal hypertension, the radius of the splenic artery could be correlated with the splenic weight, portal pressure, the grade of decrease in the portal pressure after splenectomy and the length of the splenic artery. In cases of liver fibrosis, dilatation of the splenic artery was not usually accompanied by dilatation of the proper hepatic artery, whereas in liver cirrhosis with or without giant splenomegaly, dilatation was noted not only in the splenic artery but also in the proper hepatic artery as well. - arteriogram; portal hypertension; splenic blood flow

In previous publications, we reported on the estimation of splenic blood flow in cases of portal hypertension, especially in hepatic fibrosis (Banti's syndrome) and liver cirrhosis from the histometrical determination of the radius of the splenic artery. Splenic blood flow ${ }^{1}$ was calculated therefrom by the formula of Suwa et $a l .^{2}$ It was pointed out in these reports that the splenic blood flow thus calculated was in good agreement with that obtained by dye dilution technic ${ }^{3}$ prior to splenectomy.

In the present study, the radius of the splenic artery was measured in patients with portal hypertension on the films of selective celiac arteriography and the obtained values were compared with those of direct histometrical measurement on the splenic artery after splenectomy. ${ }^{4}$ The relation of the arterial radii to portal pressure and splenic weight was investigated in an attempt to gain insight into the significance of dilatation of the splenic artery in the pathogenesis of this syndrome.

Received for publication, March 20, 1970. 
Besides, some characteristics in the estimated values of the radii of the proper hepatic arteries were investigated.

\section{Materials and Method}

Out of 153 cases of portal hypertension treated at our department, selective celiac arteriograms clear enough to permit measurement of the diameter of the splenic artery were available in 25 cases of hepatic fibrosis, 10 cases of liver cirrhosis with splenomegaly, 8 cases of liver cirrhosis and 2 cases of Budd-Chiari's syndrome, 10 cases of splenomegaly without portal hypertension (idiopathic thrombocytopenic purpura, hemolytic jaundice) and in 29 cases of control subjects. The diameter of the splenic artery was measured at the splenic hilus just proximal to the first bifurcation. The whole length of the artery was defined as the length from the branching of the splenic artery from the celiac artery to its first bifurcation at the splenic hilus. The diameter of the splenic artery measured on the angiogram was corrected in each case by the external diameter of the catheter $(2.4 \mathrm{~mm})$. Thus, the corrected radius $\left(r^{\prime}\right)$ was gained by the formula, $r^{\prime}=\frac{D \times 1.2}{K}$, where $D$ was the diameter of a lienal trunk, $K$ the diameter of a catheter. It can be assumed, therefore, that the corrected radius of the artery approximates the radius of the artery in the living body. The diameter of the hepatic artery was measured in the cases in which there was no branching of the superior measenteric artery to the liver and hepatic arterial blood was considered to be mainly supplied through the proper hepatic artery. The cases of liver cirrhosis were divided into two groups, the one with splenomegaly and the other without one. In the former were included those cases in which the splenic weight exceeded $500 \mathrm{~g}$.

\section{RESULTS}

\section{1) Arteriographic patterns of the splenic artery}

The angiographic patterns of the splenic artery in the subjects with portal hypertension and the controls can be classified into 3 groups: straight, bending and tortuous (Fig. 1). In portal hypertension, the radius of the splenic artery tended to increase with the change of arteriographic patterns from the straight to the bending or tortuous (Fig. 2). It was also noted that in the presence of portal hypertension, bending and tortuous splenic artery was more frequently encountered than in the control subjects or in cases of portal hypertension without splenomegaly. In the controls and in some patients with idiopathic thrombocytopenic purpura or hemolytic jaundice, the splenic arteries were bending but seldom tortuous. It was found that portal pressure tended to increase as the angiographic pattern of the splenic artery changed from the bending to tortuous (Fig. 3). The entire length of the artery was naturally increased in tortuous arteries than in bending ones (Fig. 4).

\section{2) The radius of the splenic artery (Fig. 5)}

In the controls, the radius of the splenic artery averaged $1.9 \mathrm{~mm}$. It was slightly increased in liver cirrhosis without splenomegaly and in splenomegaly without portal hypertension. In hepatic fibrosis, liver cirrhosis with splenomegaly and Budd-Chiari's syndrome, there was an unequivocal increase in the radius of the splenic artery. This finding is in agreement with that of the histometrical study. 

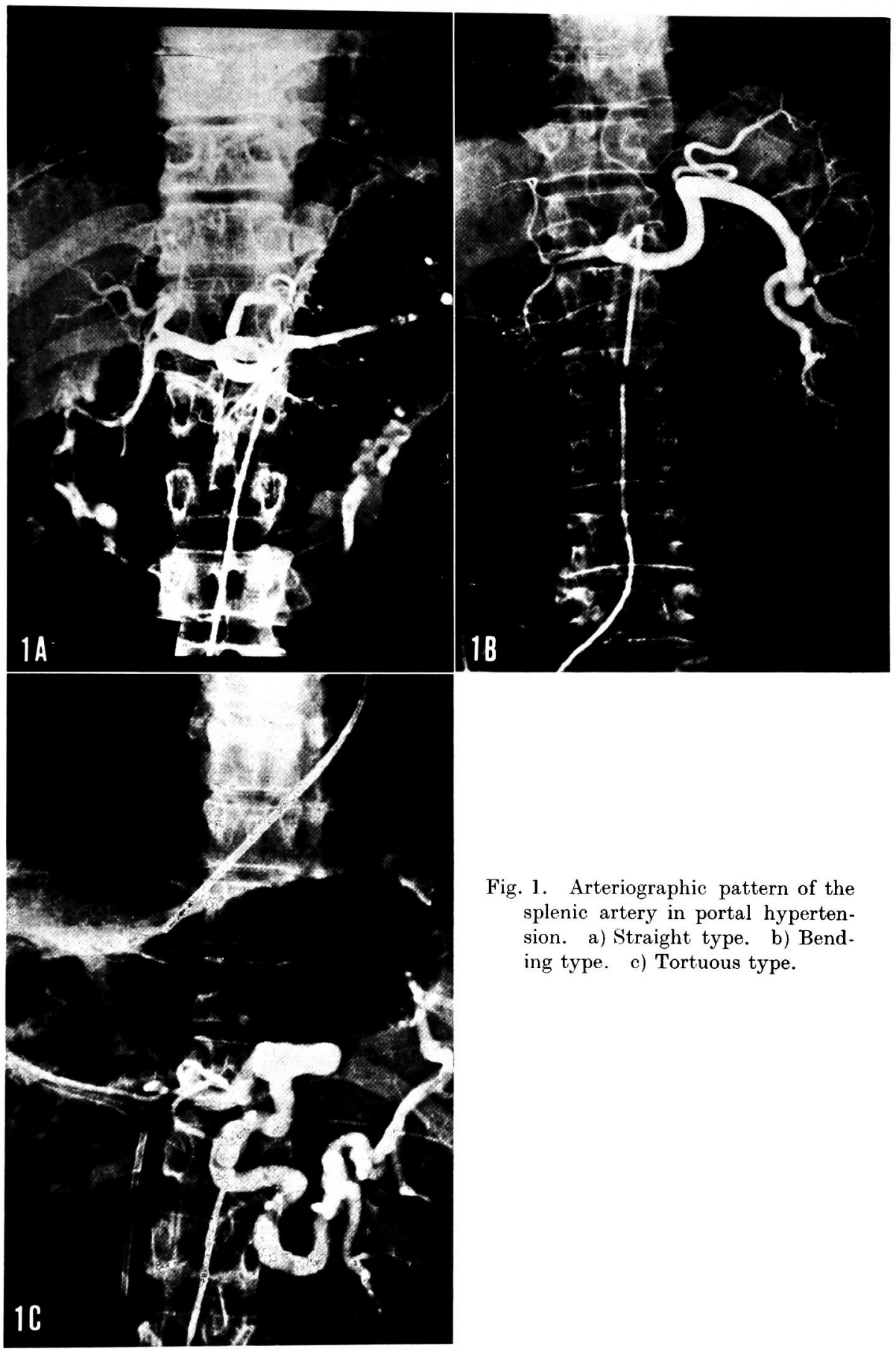

Fig. 1. Arteriographic pattern of the splenic artery in portal hypertension. a) Straight type. b) Bending type. c) Tortuous type. 


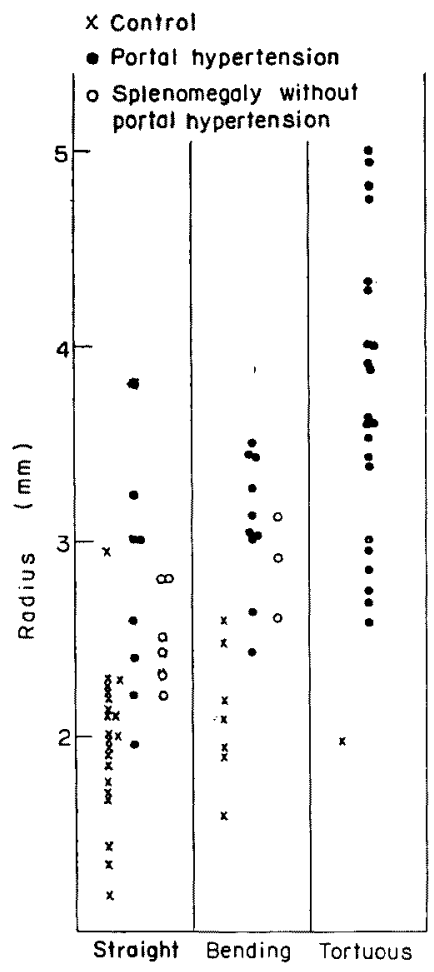

Fig. 2.

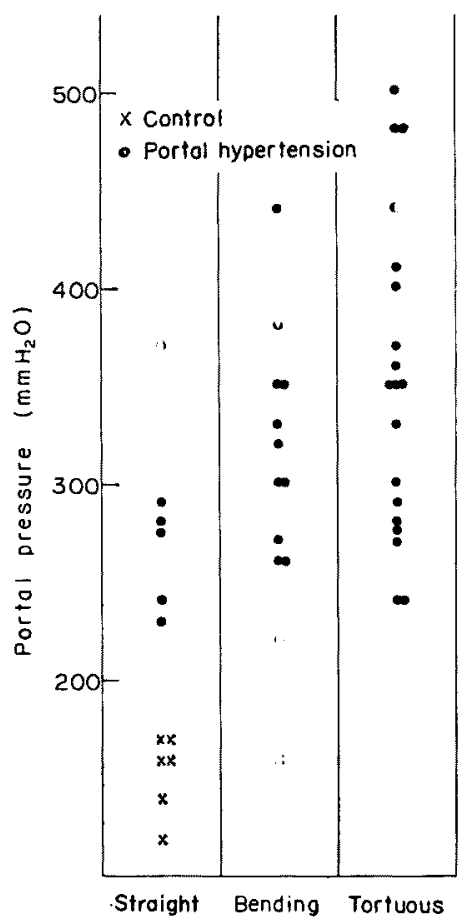

Fig. 3.

Fig. 2. Relationship between radius and angiographic pattern of the splenic artery.

Fig. 3. Relationship between portal pressure and angiographic pattern of the splenic artery.

In the majority of the cases of liver fibrosis and splenomegalic cirrhosis, the radii of the splenic arteries were measured in the same cases by both the histometrical and radiographical methods. In these cases, there was a good agreement of values obtained by the two methods. Although there was sometimes a discrepancy between the estimates by the two different methods, the average values showed the same tendency. It seems, therefore, that there is a good agreement between the radius of the artery under the influence of blood pressure in the living body and the histometrical estimate corresponding to the state of perfectly stretched internal elastic membrane. It can be stated that the splenic arterial blood flow $(Q)$ is expressed by the equation $Q=q r^{\prime n}$, where $q=5.0 \times 10^{-9} \mathrm{ml} / \mathrm{sec}, r^{\prime}=$ $\frac{D \times 1.2}{K} \times 10^{3}(\mu)$ and $n \doteqdot 2.7 . \quad D(\mathrm{~mm})$ is the diameter of the trunk of the lienal artery on arteriography, and $K(\mathrm{~mm})$, the diameter of the catheter used in arteriography. There was no difference in the radius of the splenic artery between the groups of liver fibrosis and splenomegalic cirrhosis. 


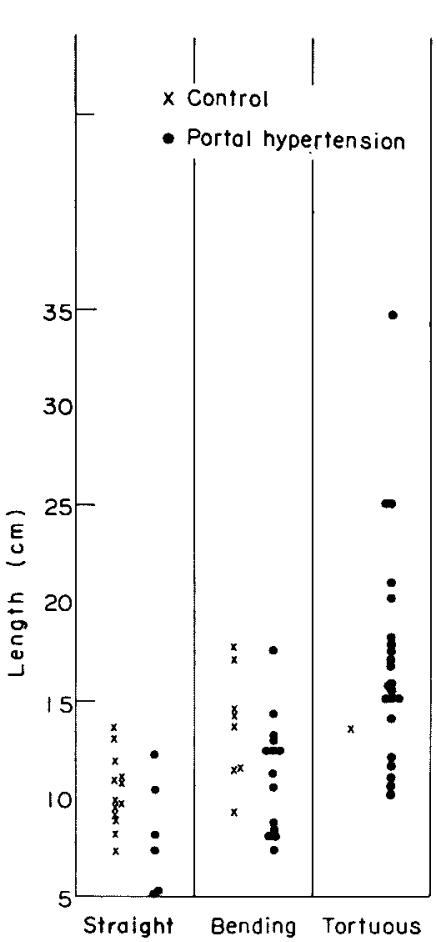

Fig. 4.

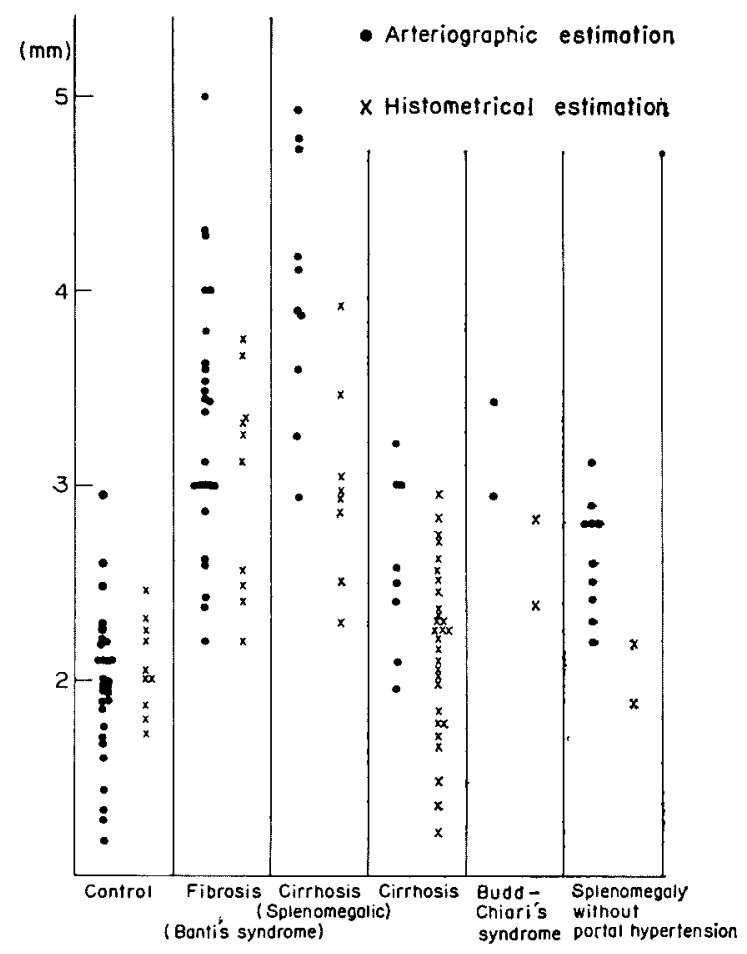

Fig. 5.

Fig. 4. Relationship between length and angiographic pattern of the splenic artery.

Fig. 5. Estimated radii of the splenic arteries in various disorders. The values obtained by histometrical and angiographical methods are in good agreement. Conspicuous increase in the radii in hepatic fibrosis and splenomegalic cirrhosis.

\section{3) Relationship between the arterial radius and splenic weight (Fig. 6)}

In the previous histometrical study a correlation was found between the arterial radius and splenic weight. In the present radiographical study a significant correlation was also ascertained with a correlation coefficient of 0.72 . In the control subjects, the splenic weight was assumed to be $100 \mathrm{~g}$, because the spleen was not removed and splenic weight could not be measured.

Besides, a correlation of the same degree was obtained, even with the cases of portal hypertension only, excluding the controls. It was also noted that the splenic artery tended to increase in radius as the splenic weight increased, regardless of the condition of the liver. The results seem to indicate a correlation between the splenic weight and splenic blood flow.

On the other hand, in idiopathic thrombocytopenic purpura, hemolytic jaundice as well as in chronic leucemia, ${ }^{1}$ dilatation of the splenic artery is not usually observed in spite of splenomegaly up to $2,000 \mathrm{~g}$. This result indicates that splenomegaly with an elevation of the portal pressure is accompanied by an 


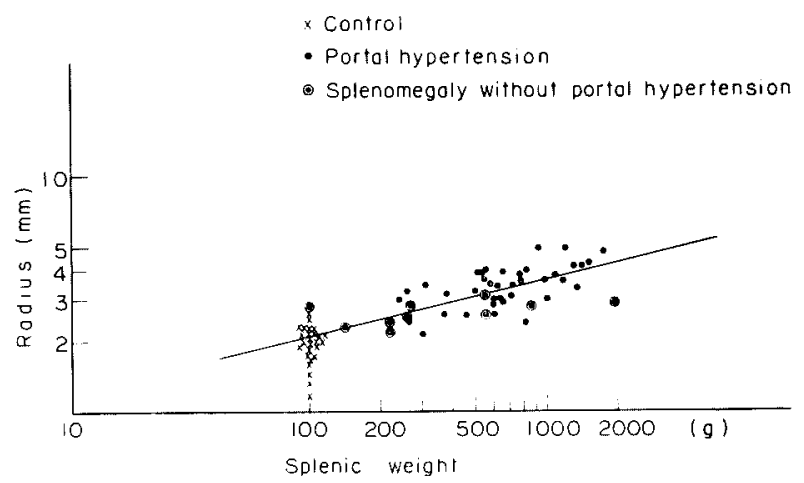

Fig. 6. A significant correlation between radius of the splenic artery and splenic weight in cases of portal hypertension. Regression equation is $Y=0.26 X+2.79$.

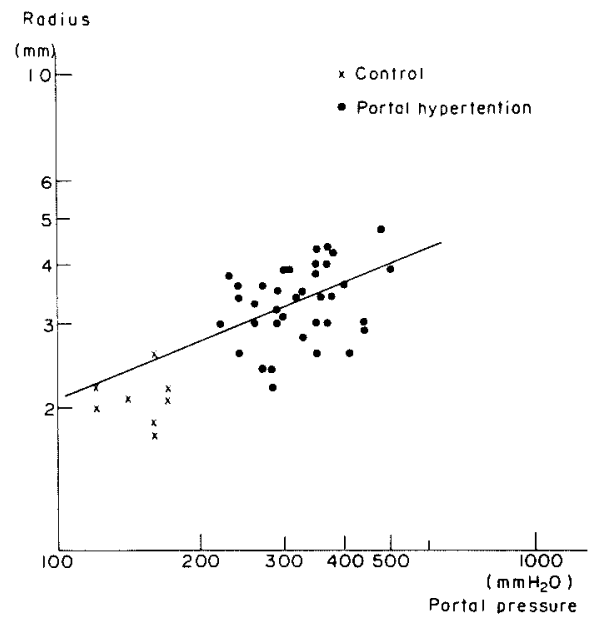

Fig. 7. A moderately significant correlation between radius of the splenic artery and portal pressure in controls and the cases of portal hypertension. $Y=0.36 X+2.6$.

increase in the splenic blood flow whereas in splenomegaly without portal hypertension the splenic blood flow is not increased. It can be inferred, therefore, that in cases of splenomegaly portal hypertension induced a rather fundamental change in the total hemodynamic aspect of the splenic circulation. In the present study of radiographic measurement of the radius of the splenic artery, there were not many cases of moderate splenomegaly under $300 \mathrm{~g}$ in splenic weight. In the cases of liver cirrhosis, the values of the radius of the splenic artery were between those in giant splenomegaly and those in the control group, as was demonstrated by the histometrical technic. 


\section{4) Relationship between the arterial radius and portal pressure (Fig. 7)}

Statistical tests prove a significant correlation between the arterial radius and portal pressure at $1 \%$ level, the correlation coefficient being 0.52 . Accordingly, elevation of portal pressure is associated with an increase in splenic blood flow.

5) Relationship between the arterial radius and portal pressure drop after splenectomy (Fig. 8)

In portal hypertension, splenectomy usually causes a decrease in the portal pressure by $50-100 \mathrm{~mm} \mathrm{H}_{2} \mathrm{O}$, although there are some cases of unchanged portal pressure even after the operation. It was observed that there was a significant correlation between the grade of the decrease in the portal pressure and the radius of the splenic artery at the level of 0.05 . In the evaluation of the effect of splenectomy, such factors as the extent of destruction of intrahepatic branches of the portal veins, the influence of blood flow in the hepatic artery and removal of extrahepatic shunts at the time of splenectomy have to be taken into account.

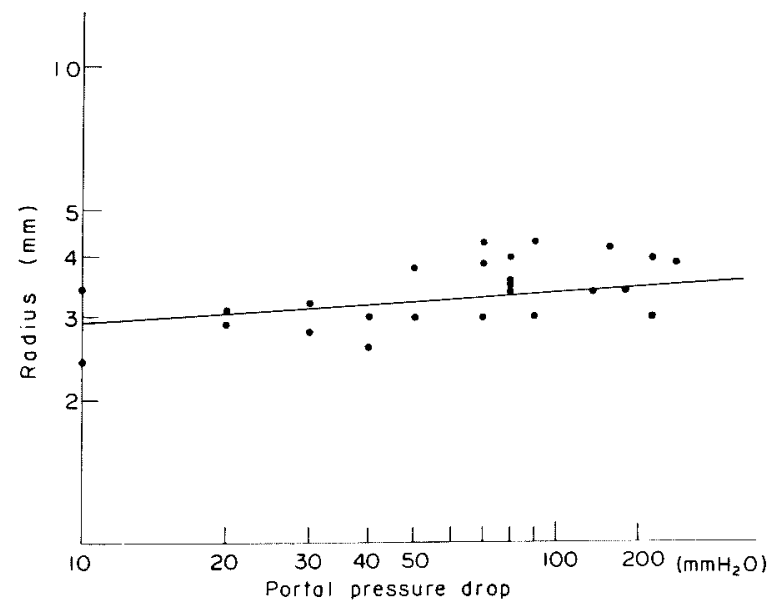

Fig. 8. Relationship between the radius of the splenic artery and portal pressure drop. $Y=0.09 X+3.38$.

There is a slight correlation between the length and diameter of the splenic artery (Fig. 9). It seems that the splenic artery is increased in length in the spleen with dilatation of the artery and an increase in the blood flow. It appears also that there is a correlation between the length of the splenic artery, the weight of the spleen and the portal pressure (Figs. 10 and 11).

\section{Discussion}

The present study revealed that the splenic blood flow as inferred from the histometrical and arteriographical estimations of the radius of the splenic artery 


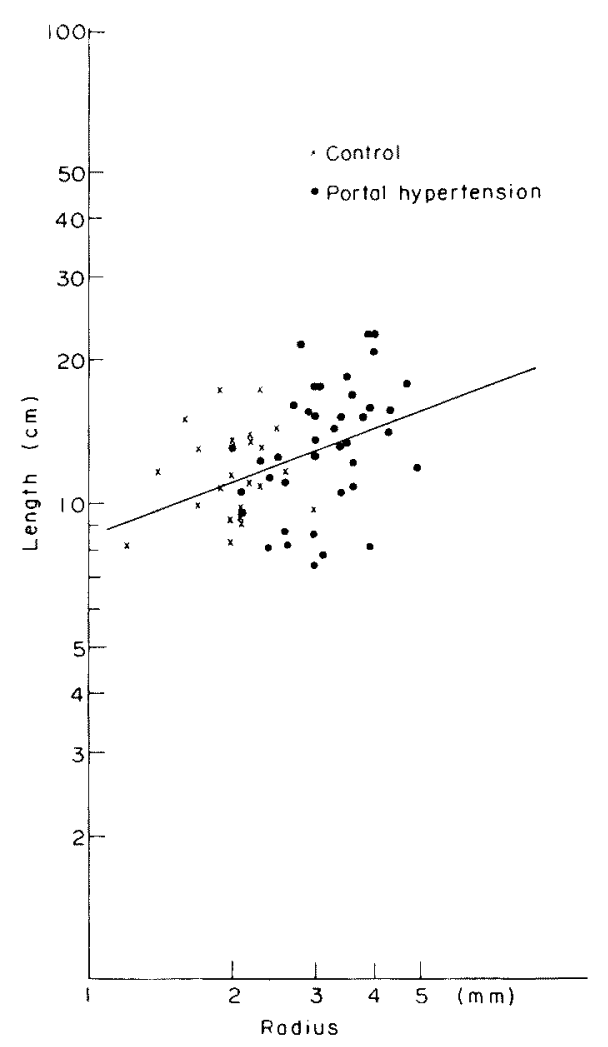

Fig. 9. Relationship between length and radius of the splenic artery.

agreed well with the values by the dye dilution method. It was thus demonstrated that the radius of the splenic artery was correlated with the splenic blood flow. Blendis et $a .^{10}$ have recently reported by measurement of the splenic artery that the splenic artery in idiopathic tropical splenomegaly with portal hypertension is dilated and that there is a positive correlation between the diameter of the splenic artery and the splenic blood flow measured with radioxenon. It seems that at least in the presence of portal hypertension dilatation of the splenic artery is an indication of increased splenic blood flow.

Liver cirrhosis with giant splenomegaly and hepatic fibrosis with splenomegaly are different from liver cirrhosis with moderate splenomegaly in that the former conditions is accompanied by an increase in the splenic blood flow. It has to be pointed out that in the cases of giant splenomegaly there is no difference in the radius of the splenic artery between liver cirrhosis and liver fibrosis.

In the cases of portal hypertension in general and in the control group, it was noted that the radius of the splenic artery increased in correlation with an increase in the splenic weight. Therefore, when two extreme conditions are considered, namely, liver cirrhosis with relatively mild splenomegaly on the one hand and 


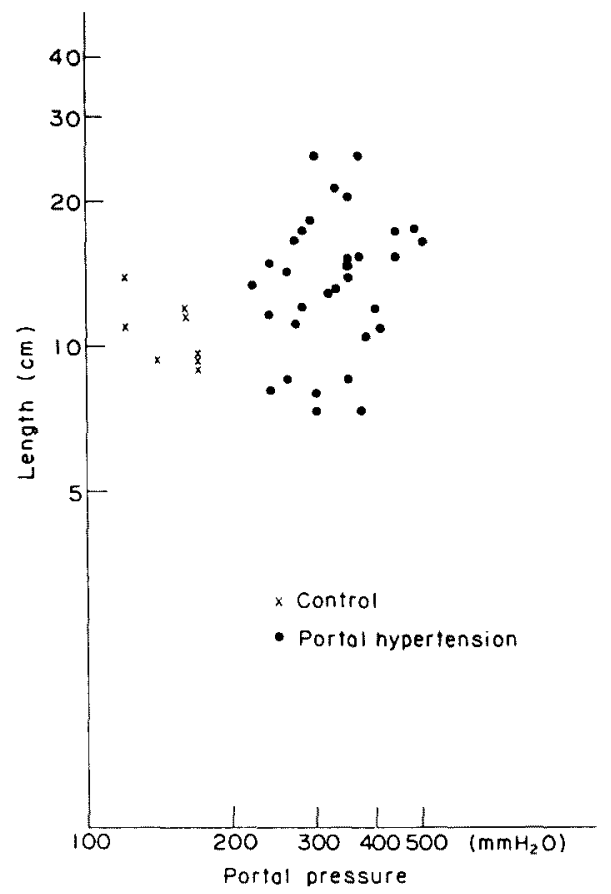

Fig. 10. Relationship between portal pressure and the length of the splenic artery.

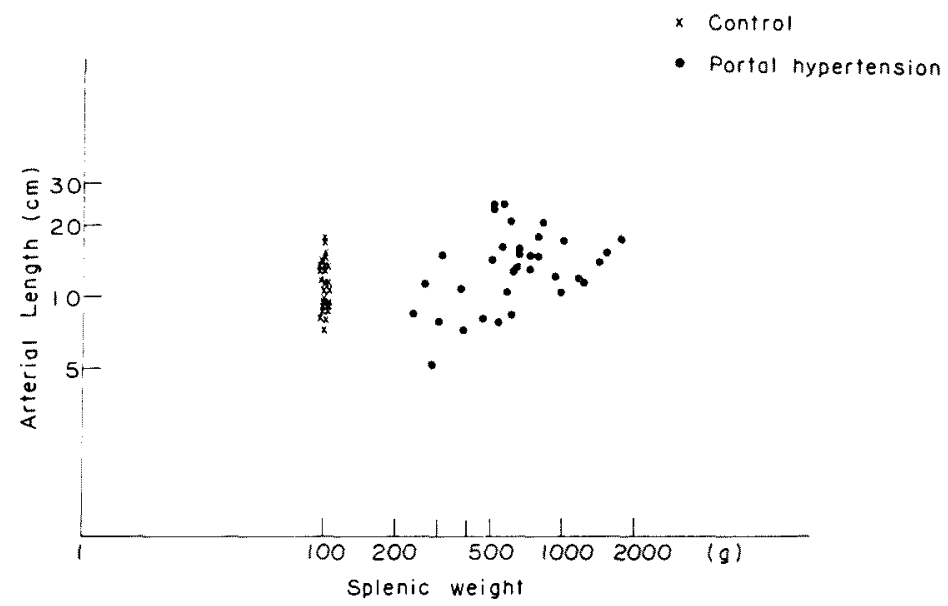

Fig. 11. Relationship between splenic weight and the length of the splenic artery.

portal hypertension with giant splenomegaly on the other, it can be stated that the increase in the radius of the splenic artery is not associated with a qualitative pathological changes of the liver. In experiments on 'high inflow portal hypertension'," it was demonstrated that an increase in splenic arterial blood flow did 
not result in an elevation of portal pressure in the absence of increased resistance in the intrahepatic portal vein. Although the dilatation of the splenic artery may play a primary role ${ }^{12}$ in the pathogenesis of splenomegaly in portal hypertension or in a further elevation of the portal pressure, the possible role of 'congestion' in inducing splenomegaly cannot be ruled out.

Nevertheless, it is evident that congestion alone cannot be responsible for the development of giant splenomegaly and advanced portal hypertension. It seems more reasonable to assume a hemodynamical condition of the spleen permitting a rise of arterial blood flow in spite of venous congestion. The assumption is substantiated by the histological and histomatrical study of Yamamoto, ${ }^{14}$ which demonstrated a transformation of the splenic cord and an increase in arteriolosinal shunts. Because arteriolosinal shunts contribute to lowering the resistance of the splenic cords to blood flow, an increase in splenic arterial blood flow is made possible even in the presence of portal hypertension.

On the other hand, we have reported on several occasions that a proliferative process of the splenic tissue precedes the development of giant splenomegaly in portal hypertension..$^{5,13}$ In portal hypertension, the increase in the splenic weight can be attributed to structural hyperplasia of the splenic cord and sinus, whereas it is mainly due to an increase in cellular elements in chronic leucemia and hemolytic jaundice. Increased splenic blood Aow seems to be possible only in association with structural hyperplasia but not with cell proliferation in the splenic cord. The influence of cell proliferation in the splenic cords upon the splenic circulation is still obscure.

As has been reported by Hales ${ }^{6}$ and Yanase, ${ }^{7}$ the radius of the proper hepatic artery is increased in a larger part of patients with liver cirrhosis (Figs. 12 and 13). In hepatic fibrosis with Banti's syndrome, dilatation of the hepatic artery is not usually found. In splenectomized liver cirrhosis, an increase in the radius of the proper hepatic artery was invariably demonstrated in the fatal cases.

There are divergent opinions in regard to the problem, whether there is a compensatory increase in the hepatic arterial blood flow in liver cirrhosis. Nakamura and Nakamura ${ }^{9}$ obtained a ratio of 43:57 for the hepatic arterial to portal blood flow in liver cirrhosis. Since the total hepatic blood flow averaged $583 \mathrm{ml} / \mathrm{min}$, the hepatic arterial blood flow amounted to $251 \mathrm{ml} / \mathrm{min}$, which was approximately the same with $239 \mathrm{ml} / \mathrm{min}$ in the control group. They obtained almost the same value in Banti's syndrome. However, Imanaga ${ }^{8}$ obtained the ratios varying from $52: 48$ to $35: 65$ and from 88:12 to $71: 29$ in liver cirrhosis and $80: 20$ in liver fibrosis, by means of their own method which allowed separate determination of arterial and portal blood flows at the time of operation. These values are quite different from those by Nakamura and suggests a compensatory increase in the blood flow through the hepatic artery. The increase in the radius of the proper hepatic artery as demonstrated in the present study suggests that the arterial blood flow is usually increased in liver cirrhosis but not in liver fibrosis because the equation $Q=q r^{\prime n}$ are considered to be valid regardless of the organs. 

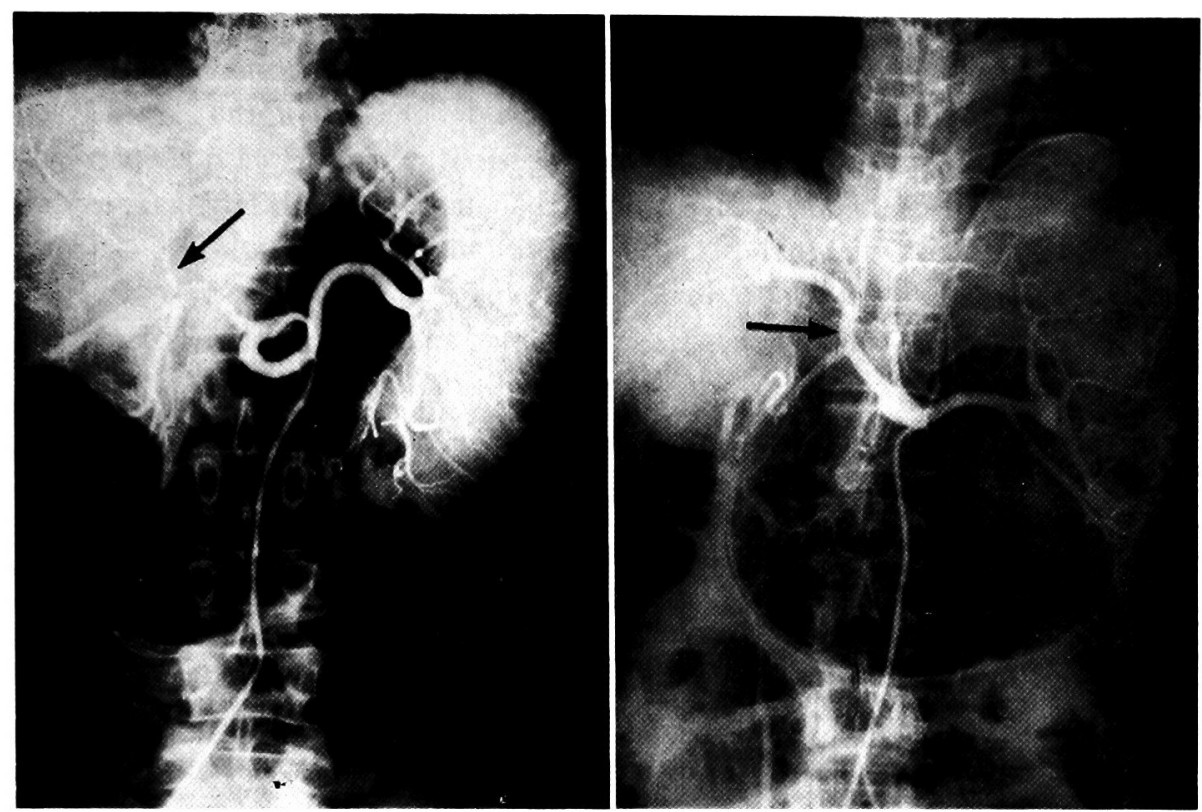

Fig. 12. One can find narrowed proper hepatic artery in hepatic fibrosis (left), compared with moderately dilated one in splenomegalic cirrhosis (right).

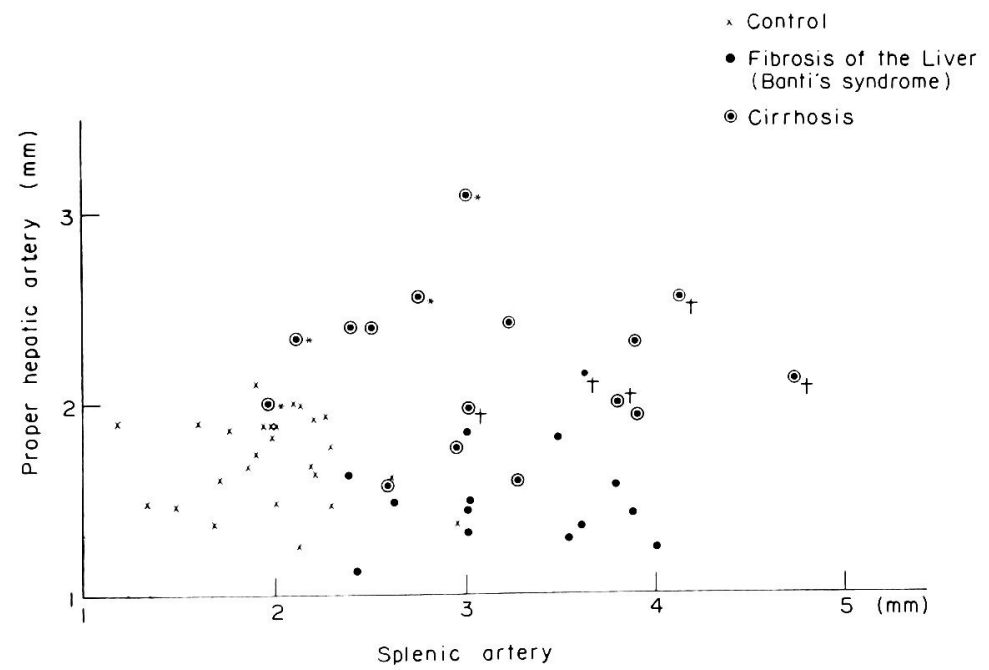

Fig. 13. Relationship between radius of the proper hepatic artery and that of the splenic artery. * Non-splenectomized, $\dagger$ Expired after splenectomy. Marked increase in the radii of the hepatic arteries in cirrhosis.

In liver cirrhosis, the larger the radius of the hepatic artery, the more injurious effect on the liver function is brought about by decreased hepatic blood flow after splenectomy. In these cases it appears that the increase in hepatic blood flow, 
which is beneficial to the maintenance of liver function, is a result of the development of the hepatic artery secondary to an increase in the intrahepatic shunts and/or to decrease in portal blood flow caused by destruction of the portal system. Blendis et $a l .^{10}$ reported that dilatation of the hepatic artery was not observed in liver cirrhosis. There are also other reports that dilatation of the hepatic artery is not always present in liver cirrhosis. ${ }^{15}$ Even in Japan, the opinions are conflicting in regard to whether the blood flow of the hepatic artery is increased in liver cirrhosis. It has to be noted, however, that in Japan liver cirrhosis is predominantly of 'postnecrotic' type, ${ }^{16}$ whereas in U.S. and European countries 'portal cirrhosis' is predominant. ${ }^{17}$ In view of this difference in the type of liver cirrhosis, it is possible that there is a certain difference in the circulatory aspect of cirrhotic livers.

\section{References}

1) Yamauchi, H. Estimation of blood flow in the Banti spleen on anatomical basis. Tohoku J. exp. Med., 1968, 95, 63-77.

2) Suwa, N., Niwa, T., Fukasawa, H. \& Sasaki, T. Estimation of intravascular blood pressure gradient by mathematical analysis of arterial casts. Tohoku J. exp. Med., $1963,79,168-198$.

3) Koyama, K. Hemodynamies of the spleen in Banti's syndrome. Tohoku J. exp. Med., 1967, 93, 199-217.

4) Furuyama, M. Histometrical investigations of arteries in reference to arterial hypertension. Tohoku J. exp. Med., 1962, 76, 388-414.

5) Seki, K. Histometrical studies of the spleen in Banti's syndrome with reference to clinicopathologic correlations. Tohoku J. exp. Med., 1965, 87, 222-243.

6) Hales, M.R., Allan, J.S. \& Hall, E.M. Injection-corrosion studies of normal and cirrhotic livers. Amer. J. Path., 1959, 35, 909-941.

7) Yanase, M. Arteriographical diagnosis of hepatic disorders. Nippon Geka Gakkai Zasshi (Jap.), 1968, 68, 1621-1651.

8) Imanaga, H. Portal Hypertension (Jap.), Kyorin Shoin, Tokyo, 1962.

9) Nakamura, T. \& Nakamura, S. Organ characteristics on vascular system in man. 2. Vascular characteristics of the liver. Tohoku Igaku Zasshi (Jap.), 1969, 77, 180-255.

10) Blendis, L., Kreel, L. \& Williams, R. The celiac axis and its branches, splenomegaly and liver diseases. Gut, 1969, 10, 85-90.

11) Sato, T., Koyama, K., Watanabe, K. \& Kimura, S. Experimental study of the effect of increase in splenic blood flow upon the portal pressure. Tohoku J. exp. Med., $1969,98,65-74$.

12) Ueda, H., Kitani, K., Kameda, H., Takeda, T., Hamada, H. \& Nagatani, Y. Idiopathic (noncirrhotic) portal hypertension (2). Naika (Jap.), 1968, 21, 733-737.

13) Sato, T., Kakizaki, G., Suda, Y. \& Saito, Y. On the pathogenesis of Banti's syndrome. Tohoku J. exp. Med., 1964, 83, 375-390.

14) Yamamoto, K. Histological studies of the terminals of the splenic artery in the normal and Banti spleens. Tohoku J. exp. Med., 1970, 101, 77-91.

15) Mclndoe, A.H. Vascular lesions of portal cirrhosis. Arch. Path., 1928, 5, 23-42.

16) Miyaji, T. Hepatoma in Japan with special reference to its relation to cirrhosis. Nippon Byori Gakkai Zasshi (Jap.), 1965, 54, 23-38.

17) Schiff, L. Diseases of the Liver, J.B. Lippincott Co., Philadelphia and Montreal, 1956 . 